\title{
TWO-DIMENSIONAL BRILLOUIN ZONES IN THIN MAGNETIC FILMS AND A MAPPING OF THE REGIONS OF EXISTENCE OF MAGNETIC SURFACE STATES
}

\author{
B. Kolodziejczak and H. Puszkarski \\ Surface Physics Division, Institute of Physics, A. Mickiewicz University \\ Matejki 48/49, 60-769 Poznań, Poland
}

\begin{abstract}
We consider the model of a thin ferromagnetic film, described by a standard Heisenberg Hamiltonian involving interactions between nearest neighbours. The surface inhomogeneities are introduced by means of "surface parameters" describing strictly the conditions at the surfaces. We consider the surface orientations $S C(111)$ and $F C C(111)$, for which we diagonalize the Hamiltonian by standard procedure obtaining the characteristic equation for the permitted eigenvalues (and eigenvectors perpendicular to the surface) versus the two-dimensional wave vector in the plane of the reciprocal lattice. It is found that the existence of a surface state (at fixed surface conditions) requires an appropriate propagation direction of the spin wave in the plane of the film. This requirement is visualized graplically by plotting the existence regions of the surface states in the two-dimensional Brillouin zone. These regions are studied as to their form and size versus the surface parameters. For the two surface orientations studied, the existence regions are found to be identical and hence they are not sensitive to the differences in cubic structure between SC and FCC.
\end{abstract}

PACS numbers: 75.30.Ds, 75.70.-i

\section{Introduction}

Typical thin film phenomena include the occurrence of states localized on the surface. The localized states themselves are a source of valuable information on the bulk properties of the system (in the case of magnetic systems their analysis can provide the values of the exchange integral and magnetization) as well as on the physical conditions on the surface (such as the surface anisotropy field). Since the surface states are sensitive to the parameters related to the surface, we can create or annihilate the surface states by changing the parameters. Unfortunately, there is a weakness of this method, namely it is very difficult to control precisely the changes in one of the parameters in a once prepared sample. There is also 
another approach to the study of the surface localized states which is the subject of the present paper. Each localized state is in fact a wave propagating in the film (in the case of magnetic films, which we shall be considering henceforth, these are spin waves). It has been proved that not only static parameters are decisive for the existence of a surface state. However, also dynamic parameters like, for example, the direction of propagation which can be easily modified in the course of investigation are important. In this work we shall study the conditions for the presence of surface states in a thin magnetic film and in particular the influence of the direction of propagation.

The present paper is a continuation of the studies of a similar system reported in Refs. [1,2]. However, the discussion presented in [2] was restricted to standing spin waves only whereas here we shall consider spin waves with an arbitrary wave vector.

\section{The model}

We consider a thin film of a ferromagnetic dielectric cut out from an arbitrary Bravais lattice along any lattice direction. Each node of the film harbours a spin interacting with its neighbours through exchange interactions. The system expands to infinity along the directions parallel to the film surface and is composed of the set of $L$ parallel, equidistant atomic planes in the direction perpendicular to the film surface. An obvious consequence of the unboundedness of the film is that spins belonging to the same lattice plane are in the same physical conditions. In our further considerations the planes will be restricted in the direction parallel to their surface by truncation and imposition of Born-Kármán periodic conditions. The system as a whole is placed in a homogeneous magnetic field $\boldsymbol{H}$. To describe this system we shall apply the classical Heisenberg model with the following Ifamiltonian:

$$
\widehat{\mathcal{H}}=-2 \sum_{\substack{l j ; l+n, j^{\prime} \\ n \leq g}} J_{n} \widehat{S}_{l j} \cdot \widehat{S}_{l+n, j^{\prime}}-g \mu_{\mathrm{B}} \sum_{l j} H_{l}^{\mathrm{eff}} \cdot \widehat{S}_{l j},
$$

where it is assumed that the summation of the scalar product of spin operators in the first term runs over the nearest neighbours of the spin denoted by $l j$. This notation means that a neighbour of the spin $l j$ lies in the plane $l+n$ at a site described by the vector $j^{\prime}$. It is moreover assumed that $n$ can take any value from 0 to a certain characteristic number $g$ which depends on the type of the lattice and the direction of the cut. This number is essential in the determination of the so-called structural functions. We shall additionally assume that the spins inside the film, that is, all spins except those in the planes 0 and $L-1$, are subject to a homogeneous effective magnetic field whereas the spins on the surfaces are moreover affected by the surface anisotropy field. The assumption that inhomogeneities occur only on the surfaces of a thin film is fundamental in the model of surface inhomogeneity (SI) within which we shall carry out our calculations.

As it has been shown, the calculations for any direction of the cut from any Bravais lattice are in general not simple. This is why we shall restrict our analysis to the type of lattice and the orientation of the direction of the cut which 
ensure that the spins nearest to the one under consideration are located at most on the neighbouring layers, labelled $l-1, l, l+1$. In the following we shall specify for which lattices and orientations this condition is fulfilled. Having reduced the Hamiltonian to diagonal form we obtain the spectrum of excitations interpreted as spin waves in the system. In the following we shall be interested only in the excitations whose energy does not fall within the band, that is, in those which correspond to the surface states. As we have already mentioned the existence of these states depends, among others, on the direction of propagation or, in terms of the reciprocal lattice, on a vector from the two-dimensional Brillouin zone. So, we shall be interested in those regions of the two-dimensional Brillouin zones in which the surface states appear.

\section{Diagonalization of the Hamiltonian}

Assuming the classical description of the spin as $S_{l j}=\gamma S$, where $\gamma$ is the unit-vector direction of quantization for all spins in the film, we can define the vector $\gamma$ as the one which minimizes the expression for the energy, obtained from the Hamiltonian by replacing the spin operators by their classical counterparts. It is then easily shown that

$$
\boldsymbol{\gamma} \|\left(\sum_{l} H_{l}^{\mathrm{eff}}\right)=L \boldsymbol{H}^{\mathrm{eff}}+\boldsymbol{K}_{\text {surf }}^{\prime}+\boldsymbol{K}_{\text {surf }}^{\prime \prime},
$$

where $\boldsymbol{K}_{\text {surf }}$ are the effective fields on the surfaces (surface anisotropy field) not related to the external field $\boldsymbol{H}$. This $(\boldsymbol{K})$ field is a phenomenological quantity responsible for the difference in the effective fields acting on the spins inside the film and on its surface. The equation given above stems from the assumptions of the SI model. Let us note that the dependence of the direction $\gamma$ (the direction of magnetization of the internal spins) on the $\boldsymbol{K}_{\text {surf }}$ is significant only for very thin films. For thicker ones, we assume that $\gamma \| H^{\text {eff }}$. Thus we can assume that the external field $\boldsymbol{H}$ and magnetization $\boldsymbol{M}$ are homogeneous in the whole film.

In consequence the effective field $H_{l}^{\text {eff }}$ inside the sample is the sum of the external field and the demagnetization field whereas on the surfaces this sum additionally includes the surface anisotropy fields

$$
\begin{aligned}
& \boldsymbol{H}_{l}^{\mathrm{eff}}=\boldsymbol{H}^{\mathrm{eff}}+\delta_{0, l} \boldsymbol{K}_{\text {surf }}^{\prime}+\delta_{l, L-1} \boldsymbol{K}^{\prime \prime}{ }_{\text {surf }}, \\
& \boldsymbol{H}^{\mathrm{eff}}=\boldsymbol{H}-\widehat{z} 4 \pi M \cos \phi,
\end{aligned}
$$

where $\phi$ is the angle between the vector $\gamma$ and $\widehat{z}$. We now proceed to solve the eigenproblem formulated by the above Hamiltonian. We shall start with some successive transformations aimed at reducing the Hamiltonian to diagonal form. The first step consists in doing transformation from the system of coordinates related to the crystal lattice, in which the direction of $\widehat{z}$ is perpendicular to the film surface, to the system in which the $\widehat{\boldsymbol{z}}^{\prime}$ axis is parallel to the direction of $\boldsymbol{\gamma}-$ the spin quantization axis. This transformation can be expressed in the form [1]

$$
\widehat{S}_{l j}=\gamma \widehat{S}_{l j}^{z^{\prime}}+\frac{1}{\sqrt{2}}\left(\boldsymbol{A} \widehat{S}_{l j}^{+}+\boldsymbol{A}^{*} \widehat{S}_{l j}^{-}\right)
$$


where the vector $\boldsymbol{A}$ is defined by the following relations:

$$
\boldsymbol{\gamma} \cdot \boldsymbol{\gamma}=1, \quad \boldsymbol{\gamma} \cdot \boldsymbol{A}=0, \quad \boldsymbol{\gamma} \times \boldsymbol{A}=\mathrm{i} \boldsymbol{A}, \quad \boldsymbol{A} \cdot \boldsymbol{A}^{*}=1, \quad \boldsymbol{A} \times \boldsymbol{A}^{*}=\mathrm{i} \boldsymbol{\gamma} .
$$

Substituting the expression for $\widehat{S}_{l j}$ into the Hamiltonian and using the above relations between $\boldsymbol{\gamma}$ and $\boldsymbol{A}$, we arrive at

$$
\begin{aligned}
& \hat{\mathcal{H}}=-2 \sum_{l j ; l+n, j^{\prime}} J_{n}\left[\widehat{S}_{l j}^{z^{\prime}} \hat{S}_{l+n, j^{\prime}}^{z^{\prime}}+\frac{1}{2}\left(\widehat{S}_{l j}^{+} \widehat{S}_{l+n, j^{\prime}}^{-}+\widehat{S}_{l j}^{-} \widehat{S}_{l+n, j^{\prime}}^{+}\right)\right] \\
& -g \mu_{\mathrm{B}} \sum_{l j}\left\{\frac{1}{\sqrt{2}}\left[\left(\boldsymbol{H}_{l}^{\mathrm{eff}} \cdot \boldsymbol{A}\right) \hat{S}_{l j}^{+}+\left(\boldsymbol{H}_{l}^{\mathrm{eff}} \cdot \boldsymbol{A}^{*}\right) \widehat{S}_{l j}^{-}\right]+\left(\boldsymbol{H}_{l}^{\mathrm{eff}} \cdot \boldsymbol{\gamma}\right) \widehat{S}_{l j}^{z^{\prime}}\right\},
\end{aligned}
$$

where the first sum originates in the Heisenberg part and the second sum - in the Zeeman part of the initial Hamiltonian.

We now apply the Holstein-Primakoff transformation using the quasi-saluration approximation. The operators $\widehat{S}_{l j}^{+}, \widehat{S}_{l j}^{-}, \widehat{S}_{l j}^{z^{\prime}}$ are replaced by appropriate combinations of the operators $\hat{a}_{l j}^{+}$and $\hat{a}_{l j}$ according to [1]

$$
\widehat{S}_{l j}^{+}=\sqrt{2 S} \widehat{a}_{l j}, \quad \widehat{S}_{l j}^{-}=\sqrt{2 S} \hat{a}_{l j}^{+}, \quad \widehat{S}_{l j}^{z^{\prime}}=S-\widehat{a}_{l j}^{+} \widehat{a}_{l j} .
$$

After some calculations we arrive at the following expression:

$$
\begin{aligned}
\widehat{\mathcal{H}}= & -2 S \sum_{l j ; l+n, j^{\prime}} J_{n}\left(\widehat{a}_{l+n, j^{\prime}}^{+} \widehat{a}_{l j}+\widehat{a}_{l j}^{+} \widehat{a}_{l+n, j^{\prime}}-\widehat{a}_{l j}^{+} \widehat{a}_{l j}-\widehat{a}_{l+n, j^{\prime}}^{+} \widehat{a}_{l+n, j^{\prime}}\right) \\
& +g \mu_{\mathrm{B}} \sum_{l j}\left(H_{l}^{\text {eff }} \cdot \gamma\right) \widehat{a}_{l j}^{+} \widehat{a}_{l j},
\end{aligned}
$$

where only the terms with $a_{l j}$ operators in the second power are preserved. The terms of zero order with respect to these operators are responsible only for a shift on the energy scale, which is irrelevant in our considerations, and the terms linear in these operators cancel out if the $\gamma$ vector is required to have the direction for which the total energy is minimum [1]. The terms of the fourth order with respect to $a_{l j}$ are responsible for the interactions among magnons. As these effects are beyond our interest in this paper (restricted to the low-temperature approximation) we can disregard these terms. In spite of these substantial simplifications, our IIamiltonian is still very complex. Thus, to simplify the problem further, we have recourse to the translational invariance in directions parallel to the film surface. In view of this, the assumption of infinite expansion of the film can be replaced by the equivalent truncation of the system and imposition of Born-Kármán periodic boundary conditions, with the additional assumption that each plane contains $N$ spins. The procedure is carried out in a third transformation - a Fourier transformation in the plane of the film, according to [1]

$$
\begin{aligned}
& \widehat{a}_{l j}^{+}=\frac{1}{\sqrt{N}} \sum_{k_{\| l}} \exp \left(\mathrm{i} k_{\| l} \cdot j\right) \widehat{b}_{k_{\|} l}^{+}, \\
& \widehat{a}_{l j}=\frac{1}{\sqrt{N}} \sum_{k_{\|}} \exp \left(-\mathrm{i} k_{\|} \cdot j\right) \widehat{b}_{k_{\|} l},
\end{aligned}
$$


where $k_{\|}$is the vector of the reciprocal lattice from the two-dimensional Brillouin zone corresponding to the Bravais lattice in the plane. The outcome of this procedure is

$$
\begin{aligned}
\widehat{\mathcal{H}}= & \sum_{k_{i l}}\left\{\sum_{l=0}^{g-1} \sum_{n=-l}^{g} R_{l, l+n}\left(k_{\|}\right) \widehat{b}_{k_{\| l} l}^{+} \widehat{b}_{k_{\|} l+n}+\sum_{l=g}^{L-1-g} \sum_{n=-g}^{g} R_{l, l+n}\left(k_{\|}\right) \widehat{b}_{k_{\|} l}^{+} \widehat{b}_{k_{\|} l+n}\right. \\
& \left.+\sum_{l=L-g}^{L-1} \sum_{n=g}^{L-1-l} R_{l, l+n}\left(k_{\|}\right) \widehat{b}_{k_{\| l} l}^{+} \widehat{b}_{k_{\|} l+n}\right\}
\end{aligned}
$$

where $R_{l, l+n}\left(k_{\|\|}\right)$is of the form

$$
R_{l, l+n}\left(k_{\|}\right)=-2 S J_{n} \Gamma_{n}^{k_{\|}}+\delta_{0, n}\left[2 S \sum_{m=-g}^{g} J_{m} z_{m}+g \mu_{\mathrm{B}}\left(H_{l}^{\mathrm{eff}} \cdot \gamma\right)\right] .
$$

The new symbols introduced above are defined as follows. The structural factor $\Gamma_{n}^{k} \|$ is defined by

$$
\Gamma_{n}^{ \pm k_{\|}} \equiv \sum_{j^{\prime}} \exp \left[ \pm i k_{\|} \cdot\left(j-j^{\prime}\right)\right]
$$

where summation over $j^{\prime}$ runs over all nearest neighbours of the spin in the $l$ plane of the $j$ vector (arbitrary but fixed values) hosted in the $l+n$ plane and described by the appropriate $j^{\prime}$ vectors; the $\Gamma_{n}^{k_{\| 1}}$ satisfy the relations

$$
\Gamma_{-n}^{k_{\|}}=\left(\Gamma_{n}^{k_{\|}}\right)^{*}=\Gamma_{n}^{-k_{\|}}, \quad \Gamma_{0}^{k_{\|}}=\left(\Gamma_{0}^{k_{\|}}\right)^{*} .
$$

The parameter $z_{n} \equiv \Gamma_{n}^{0}$ denotes the number of spins which are neighbours of a spin belonging to the plane $l$ (located in the planes $l \pm n$ ). The number $g$ appearing in the limits of summation signifies that the nearest neighbours of a given spin from the plane $l$ are located in the planes which are at most at a distance $g$ from the plane $l$, so that they lie in the planes between $l-g$ and $l+g$ with these two planes included. This number $g$ depends on the type of the lattice and on the direction of the film cut $[4,5]$.

It is worth noting that all the information on the structure of the lattice from which the thin film has been cut is included entirely in the structural factors $\Gamma_{n}^{k_{\|}}$. Since our Hamiltonian is not yet diagonalized, we shall perform the last transformation perpendicular to the plane of the surface [1]. Assuming that the operators $\widehat{b}_{k_{\|} l}^{+}$and $\widehat{b}_{k_{\|} l}$ can be expressed as linear combinations of new operators $\widehat{\xi}$ and $\hat{\xi}^{+}$

$$
\hat{b}_{k_{\| l} l}^{+}=\sum_{\tau} u_{i}^{*}(\tau) \hat{\xi}_{k_{\| l} \tau}^{+}, \quad \widehat{b}_{k_{\| l} l}=\sum_{\tau} u_{i}(\tau) \hat{\xi}_{k_{\|} \tau},
$$

with the requirement that the functions $u_{l}(\tau)$ take a form such that the Hamiltonian written in terms of the $\widehat{\xi}_{k_{\| l} l}$ operators shall be diagonal, i.e., of the form

$$
\widehat{\mathcal{H}}=\sum_{k_{\|} \tau} E\left(k_{\|\|}, \tau\right) \widehat{\xi}_{k_{\|} \tau}^{+} \widehat{\xi}_{k_{\|} \tau}
$$


and imposing the orthogonality conditions:

$$
\sum_{l} u_{l}(\tau) u_{l}^{*}\left(\tau^{\prime}\right)=\delta_{\tau \tau^{\prime}}, \quad \sum_{\tau} u_{l}(\tau) u_{l^{\prime}}^{*}(\tau)=\delta_{l l^{\prime}},
$$

we arrive at the following set of difference equations

$$
u_{l}(\tau) E\left(k_{\|}, \tau\right)=\sum_{n=-g}^{g} R_{l, l+n}\left(k_{\|}\right) u_{l+n}(\tau), \quad l=0,1, \ldots, L-1,
$$

which may also be expressed in the form

$$
\begin{gathered}
{\left[R\left(k_{\|}\right)-E\left(k_{\|}, \tau\right)-a_{l}\right] u_{l}(\tau)-2 S \sum_{\substack{n=-g \\
n \neq 0}}^{g} J_{n} \Gamma_{n}^{k_{\|}} u_{l+n}(\tau)=0} \\
l=0,1, \ldots, L-1,
\end{gathered}
$$

where the symbols $R\left(k_{\|}\right)$and $a_{l}$ are defined as

$$
\begin{aligned}
& R\left(k_{\|}\right)=-2 S J_{0} \Gamma_{0}^{k_{\|} \|}+2 S \sum_{m=-g}^{g} J_{m} z_{m}+g \mu_{\mathrm{B}}\left(H^{\mathrm{eff}} \cdot \gamma\right), \\
& a_{0}=a=2 S \sum_{m=1}^{g} J_{m} z_{m}-g \mu_{\mathrm{B}}\left(\boldsymbol{K}_{\text {surf }}^{\prime} \cdot \boldsymbol{\gamma}\right), \\
& a_{L-1}=b=2 S \sum_{m=1}^{g} J_{m} z_{m}-g \mu_{\mathrm{B}}\left(K^{\prime \prime}{ }_{\text {surf }} \cdot \gamma\right) \\
& a_{i}=a_{L-1-i}=2 S \sum_{m=i+1}^{g} J_{m} z_{m}, \quad i=1, \ldots, g-1, \\
& a_{l}=0, \quad l=g, \ldots, L-1-g .
\end{aligned}
$$

The parameters $a_{i}$ convey the influence of the surface conditions inside the film. Since the solution of this problem in such a general form still poses much difficulty, in the following we shall only be interested in systems for which $g \leq 1$. As follows from [5], for cubic lattices this condition is fulfilled only in the following seven cases:

$$
\begin{array}{ll}
\text { simple cubic lattice } & \text { cut }\{100\} \text {, cut }\{110\}, \text { cut }\{111\} ; \\
\text { face-centred cubic lattice } & \text { cut }\{100\} \text {, cut }\{111\} ; \\
\text { body-centred cubic lattice } & \text { cut }\{100\}, \text { cut }\{110\} .
\end{array}
$$

Since for such systems the respective matrix is at most three-diagonal, we can apply the procedure presented in [1] which allows us to get the so-called characteristic equation. 


\section{The characteristic equation}

As it follows from the preceding discussion the relevant eigenproblem can be expressed in the form

$$
\begin{aligned}
& {\left[R\left(k_{\|}\right)-E\left(k_{\|}, \tau\right)-a\right] u_{0}(\tau)-2 S J_{1} \Gamma_{1}^{k_{\|}} u_{1}(\tau)=0} \\
& -2 S J_{1}\left(\Gamma_{1}^{k_{\|}}\right)^{*} u_{l-1}(\tau)+\left[R\left(k_{\|}\right)-E\left(k_{\|}, \tau\right)\right] u_{l}(\tau)-2 S J_{1} \Gamma_{1}^{k_{\|}} u_{l+1}(\tau)=0 \\
& \quad l=1, \ldots, L-2 \\
& -2 S J_{1}\left(\Gamma_{1}^{k_{\|}}\right)^{*} u_{L-2}(\tau)+\left[R\left(k_{\|}\right)-E\left(k_{\|}, \tau\right)-b\right] u_{L-1}(\tau)=0
\end{aligned}
$$

where we have employed the relation (14) for $\Gamma_{n}^{k_{\|}}$, and the other parameters appearing in the above formulae are given by

$$
\begin{aligned}
& R\left(k_{\|}\right)=-2 S J_{0} \Gamma_{0}^{k_{\|}}+2 S J_{0} z_{0}+2 S J_{1} z_{1}++g \mu_{\mathrm{B}}\left(\boldsymbol{H}^{\mathrm{eff}} \cdot \gamma\right), \\
& a=2 S J_{1} z_{1}-g \mu_{\mathrm{B}}\left(\boldsymbol{K}_{\text {surf }}^{\prime} \cdot \boldsymbol{\gamma}\right), \\
& b=2 S J_{1} z_{1}-g \mu_{\mathrm{B}}\left(\boldsymbol{K}^{\prime \prime}{ }_{\text {surf }} \cdot \boldsymbol{\gamma}\right) .
\end{aligned}
$$

The above set of equations is found to be identical to that given in Ref. [1]. Thus, we can apply the method developed therein. Introducing fictitious planes labelled $l=-1$ and $l=L$ we rewrite our set of equations as follows:

$$
\begin{gathered}
-2 S J_{1}\left(\Gamma_{1}^{k_{\|}}\right)^{*} u_{l-1}(\tau)+\left[R\left(k_{\|}\right)-E\left(k_{\|}, \tau\right)\right] u_{l}(\tau)-2 S J_{1} \Gamma_{1}^{k_{\|}} u_{l+1}(\tau)=0, \\
l=0,1, \ldots, L-1
\end{gathered}
$$

and the new boundary conditions as

$$
A_{1} u_{0}(\tau)=\frac{1}{z_{1}}\left(\Gamma_{1}^{k_{\|}}\right)^{*} u_{1}(\tau), \quad A_{2} u_{L-1}(\tau)=\frac{1}{z_{1}} \Gamma_{1}^{k_{\|}} u_{L}(\tau)
$$

where

$$
\begin{aligned}
& A_{1} \equiv \frac{a}{2 S z_{1} J_{1}}=1-\frac{g \mu_{\mathrm{B}}}{2 S z_{1} J_{1}}\left(K_{\text {surf }}^{\prime} \cdot \gamma\right), \\
& A_{2} \equiv \frac{b}{2 S z_{1} J_{1}}=1-\frac{g \mu_{\mathrm{B}}}{2 S z_{1} J_{1}}\left(K^{\prime \prime}{ }_{\text {surf }} \cdot \gamma\right)
\end{aligned}
$$

with $z_{1}$ - the number of nearest neighbours in the neighbouring plane. Let us assume that the solutions of equations of this type can be written in the form

$$
u_{l}(\tau)=\mathrm{e}^{\mathrm{i} r l}, \quad \tau \in \mathcal{C} .
$$

Inserting the above defined solutions into the equation (31) we obtain the following dispersion relation (the imaginary part of $\Gamma_{1}^{k_{11}}$ is incorporated into $\tau-$ see [1]):

$$
E\left(k_{\|}, \tau\right)=2 S J_{0}\left(z_{0}-\Gamma_{0}^{k_{\|} \|}\right)+4 S J_{1}\left(z_{1}-\left|\Gamma_{1}^{k_{\|}}\right| \cos \tau\right) .
$$

Omitting the constant terms, assuming $J_{1}=J_{0}=J$, Eq. (36) can be expressed as

$$
E\left(k_{\|}, \tau\right)=-\Gamma_{0}^{k_{\|}}-2\left|\Gamma_{1}^{k_{\|}}\right| \cos \tau
$$


with an accuracy to a constant factor. As the energy of the surface states lics outside the band, the number $\tau$, which is the perpendicular component of the wave vector, must be complex, and, to be more exact, it has to be expressible as

$$
\begin{array}{ll}
\tau=\mathrm{i} t, & \text { where } t \in\langle 0, \infty\rangle \quad \text { or } \\
\tau=\pi-\mathrm{i} \ell, & \text { where } t \in\langle 0, \infty\rangle .
\end{array}
$$

In this work we shall consider only the first possibility which corresponds to acoustic surface states. At this point we can start to derive the relationship determining the allowed values of $\tau$ is known as the characteristic equation. After Ref. [2], we insert the solution expressed as

$$
u_{l}(\tau)=C_{1} \cos \left(\frac{L-1}{2}-l\right) \tau+C_{2} \sin \left(\frac{L-1}{2}-l\right) \tau
$$

into the equations for the boundary conditions, thus obtaining a set of two homogeneous equations with two unknowns $C_{1}$ and $C_{2}$

$$
\begin{aligned}
& {\left[\alpha \cos \frac{L-1}{2} \tau-\cos \frac{L+1}{2} \tau\right] C_{1}+\left[\alpha \sin \frac{L-1}{2} \tau-\sin \frac{L+1}{2} \tau\right] C_{2}=0,} \\
& {\left[\beta \cos \frac{L-1}{2} \tau-\cos \frac{L+1}{2} \tau\right] C_{1}+\left[-\beta \sin \frac{L-1}{2} \tau-\sin \frac{L+1}{2} \tau\right] C_{2}=0,}
\end{aligned}
$$

where, for the sake of simplicity, we have introduced new coefficients $\alpha$ and $\beta$ defined as

$$
\alpha=\frac{A_{1}\left|\Gamma_{1}^{0}\right|}{\left|\Gamma_{1}^{k_{\|}}\right|}, \quad \beta=\frac{A_{2}\left|\Gamma_{1}^{0}\right|}{\left|\Gamma_{1}^{k_{\|}}\right|} .
$$

The above set has nontrivial solutions if and only if its determinant is zero, therefore

$$
(\alpha+\beta) \sin L \tau-\alpha \beta \sin (L-1) \tau-\sin (L+1) \tau=0 .
$$

Introducing new parameters defined as [2]

$$
r=\frac{\alpha \beta-1}{\alpha \beta+1}, \quad p=\frac{\alpha+\beta}{\alpha \beta+1},
$$

after some transformations we arrive at the desired characteristic equation

$$
\tan L \tau=r \frac{\sin \tau}{\cos \tau-p} .
$$

Moreover, skipping some tedious calculations, we obtain the following equation for $u[2]$

$$
\begin{aligned}
u_{l}(\tau) & =C(\alpha, \tau)\left[\alpha_{s}(\alpha, \tau) \cos \left(\frac{L-1}{2}-l\right) \tau\right. \\
& \left.-\alpha_{c}(\alpha, \tau) \sin \left(\frac{L-1}{2}-l\right) \tau\right]
\end{aligned}
$$

where the functions $C(\alpha, \tau), \alpha_{c}(\alpha, \tau)$ and $\alpha_{s}(\alpha, \tau)$ are given by

$$
\begin{aligned}
& C(\alpha, \tau)=\sqrt{2}\left[\left(L+\frac{\sin L \tau}{\sin \tau}\right) \alpha_{s}^{2}(\alpha, \tau)+\left(L-\frac{\sin L \tau}{\sin \tau}\right) \alpha_{c}^{2}(\alpha, \tau)\right]^{-\frac{1}{2}}, \\
& \alpha_{s}(\alpha, \tau)=\sin \frac{L+1}{2} \tau-\alpha \sin \frac{L-1}{2} \tau
\end{aligned}
$$




$$
\alpha_{c}(\alpha, \tau)=\cos \frac{L+1}{2} \tau-\alpha \cos \frac{L-1}{2} \tau .
$$

Taking into account that $\tau=\mathrm{i}$ we obtain

$$
\begin{aligned}
& \tanh L \iota=r \frac{\sinh t}{\cosh t-p}, \\
& u_{l}^{A}(t)=D(\alpha, t)\left[\beta_{s}(\alpha, t) \cosh \left(\frac{L-1}{2}-l\right) t\right. \\
& \left.-\beta_{c}(\alpha, t) \sinh \left(\frac{L-1}{2}-l\right) t\right], \\
& D(\alpha, t)=\sqrt{2}\left[\left(L+\frac{\sinh L t}{\sinh t}\right) \beta_{s}^{2}(\alpha, t)+\left(L-\frac{\sinh L t}{\sinh t}\right) \beta_{c}^{2}(\alpha, t)\right]^{-\frac{1}{2}}, \\
& \beta_{s}(\alpha, t)=\sinh \frac{L+1}{2} t-\alpha \sinh \frac{L-1}{2} t, \\
& \beta_{c}(\alpha, t)=\cosh \frac{L+1}{2} t-\alpha \cosh \frac{L-1}{2} t .
\end{aligned}
$$

It is worth noting that the characteristic equation depends only on the parameters $r, p$ and $L$. Knowing what type of solutions we are looking for we can try to determine for which values of these parameters the required solutions are obtainable. Such a procedure was performed in Ref. [2] and led to the so-called pinning diagram, presented in Fig. 1, which enables us to predict the types of states realizable in our model for given values of $r, p$ and $L$.

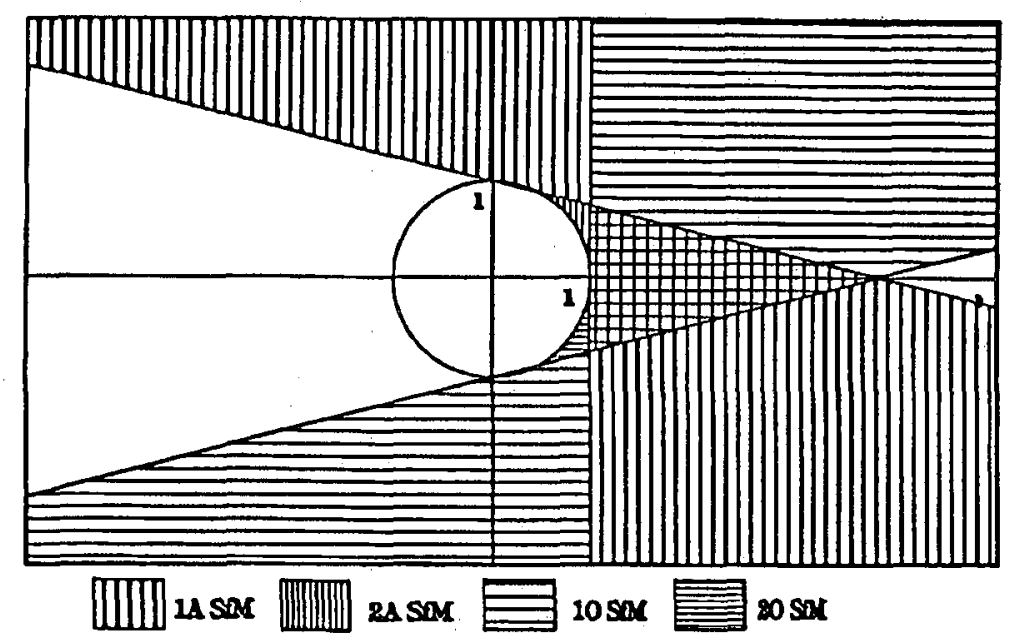

Fig. 1. Pinning diagram. In the plane of parameters $r, p$ one distinguishes regions in which there exists 1 or 2 acoustic surface modes (ASfM), 1 or 2 optical surface modes (OSfM), and no surface mode (unshaded area - except the interior of the circle which does not have physical meaning). 


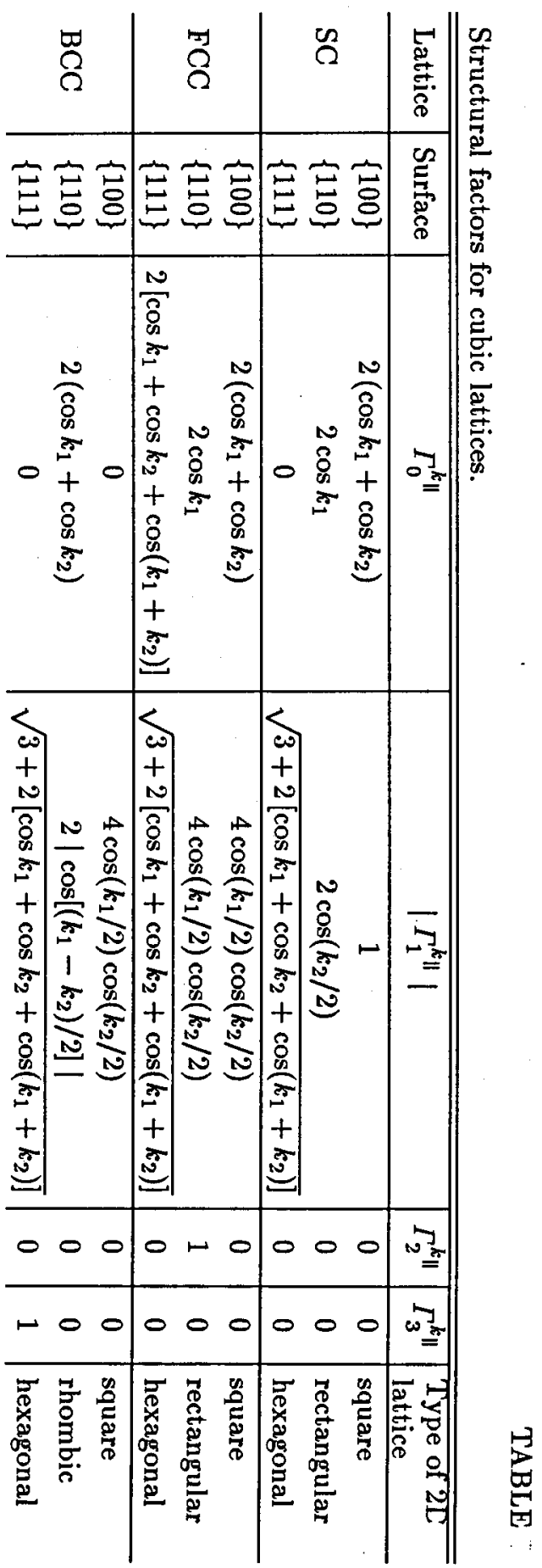


Since the parameters $r$ and $p$ are dependent on $\alpha$ and $\beta$ which in turn are related through $\Gamma_{1}^{k} \|$ with $k_{\|}$, the latter parameter will affect the appearance and disappearance of the surface states in our model. In the following we shall concentrate on the changes in structure of the energy spectrum induced by changes in the vector $k_{\|}$and shall restrict our considerations to certain fixed paths on the Brillouin zone.

\section{Structural functions}

As follows from the preceding analysis, the behaviour of the system is very strongly affected by the functions $\Gamma_{1}^{k \|}$ which, for fixed values of the parameters $A_{1}, A_{2}$ and $L$, decide about the energy of the state, the width of the band and the existence and type of the surface states. Therefore it is of interest to know the explicit form of these functions. Their derivation was the subject of Refs. [4] and [5] and the results obtained therein are quoted in Table (the values of $k_{1}$ and $k_{2}$ used in the table are components of $k_{i \|}$ in the coordinate system of unit vectors of the reciprocal lattice). Although we had intended to consider only the cases for which $g \leq 1$, for the sake of completeness the table includes also the cases FCC $\{110\}$ and BCC $\{111\}$ for which this condition is not satisfied.

The function $\Gamma_{0}^{k_{\|}}$is a factor modulating the energy spectrum but not affecting the structure of the spectrum so that its presence will be of no particular importance in our analysis. According to the above discussion it is the function $\left|\Gamma_{1}^{k_{\|}}\right|$which exerts the decisive influence on the behaviour of our model as only this function appears indirectly in the expressions for $r$ and $p$ in the characteristic equation. With the help of the pinning diagram and the explicit expression for $\left|\Gamma_{1}^{k_{\|}}\right|$we can pinpoint the regions where the localized states appear on the Brillouin zone taking advantage of the fact that the borderlines between the areas of their occurrence on the zone are the cross-section lines (isohypses) of this structural function. Moreover, it should be remembered that the function $\left|\Gamma_{1}^{\boldsymbol{k}_{\|}}\right|$ in its explicit form influences only the dynamics and the region of appearance of the localized states but the variation of $\left|\Gamma_{1}^{k_{\|} \mid}\right|$from its maximum to its minimum value (zero) does not affect the structure of the energy spectrum as a whole. In view of this, we shall not study functions $\left|\Gamma_{1}^{k_{\|}}\right|$corresponding to different crystal structures, but shall focus our attention on one of them which brings interesting results. To be more precise we shall analyze the structures SC $\{111\}$ and FCC $\{111\}$ for a two-dimensional hexagonal lattice when the structural function $\left|\Gamma_{1}^{k} \|\right|$ takes the form

$$
\left|\Gamma_{1}^{k_{\|}}\right|=\sqrt{3+2\left[\cos k_{1}+\cos k_{2}+\cos \left(k_{1}+k_{2}\right)\right]} .
$$

The surface corresponding to the above function, Eq. (53), spanned over the zone, is shown in Fig. 2. As we want to examine the changes in the energy spectrum induced by the changes in the vector $k_{\|}$, we have chosen a path of changes of this vector on the Brillouin zone (Fig. 3). Figure 4 illustrates the changes in $\left|\Gamma_{1}^{k_{\|}}\right|$ corresponding to the changes of $k_{\|}$along the path marked in Fig. 3, from which 


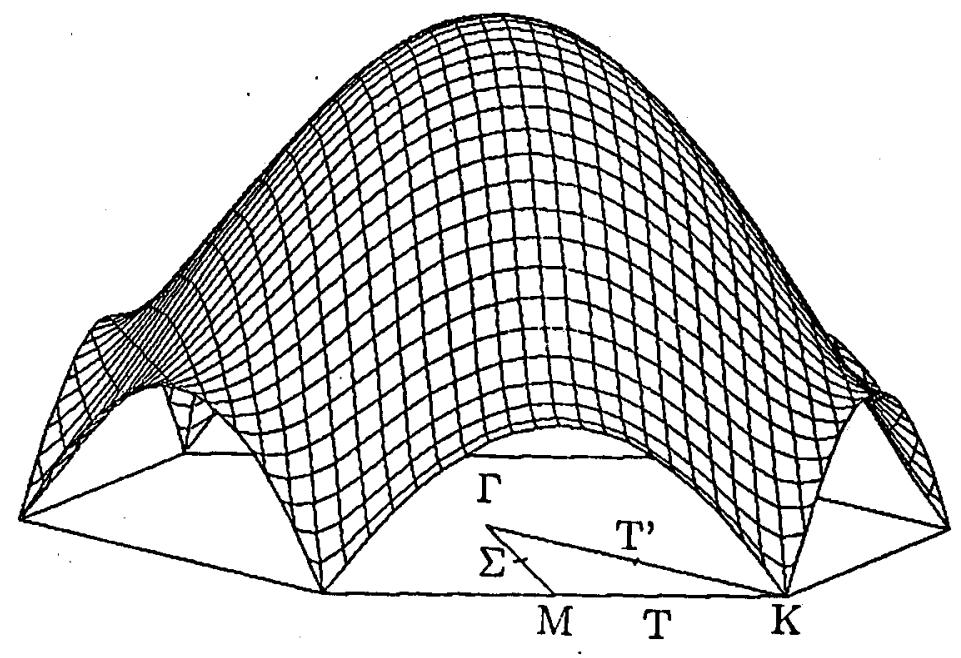

Fig. 2. The surface of the function $\left|\Gamma_{1}^{k} \|\right|$ within the Brillouin zone, for SC $\{111\}$ structure.
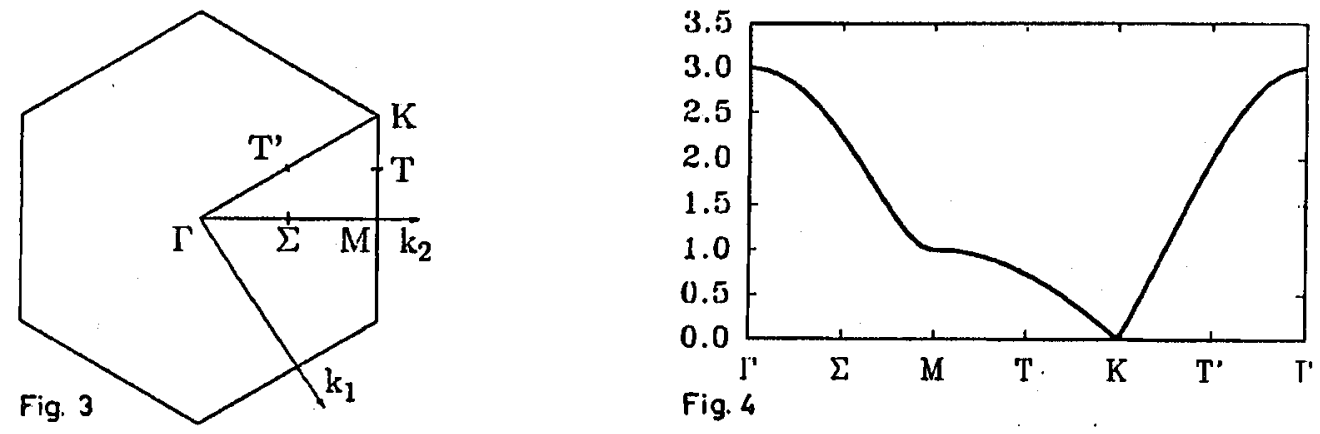

Fig. 4

Fig. 3. A selected path of variations of the in-plane wave vector $k_{\|}$(n the 1st Brillouin zone for SC $\{111\}$ structure with the characteristic points of the zone indicated thereon. Fig. 4. Graph of the function $\left|\Gamma_{1}^{k} \|\right|$ versus $k_{\|}$as the latter varies along the path shown in Fig. 3.

we can draw quantitative and not only qualitative (as in the case illustrated in Fig. 2) conclusions.

\section{The surface states}

In thin film systems the formation of a localized state on the surface (surface state) can be induced by choosing appropriate values of the surface parameters denoted by $A_{1}$ and $A_{2}$. This fact has been used in investigations of spin wave resonance where standing waves are considered [1]. The same result is achieved (as we shall show) through a change in the dynamic variable, the wave vector 
$k_{\|}$. This technique cannot be used in spin wave resonance where $k_{\|}=0$ and, instead, methods permitting the observation of travelling waves, e.g. Brillouin light scattering, should be used. A theoretical confirmation of the influence of the vector $k_{\|}$on the generation of surface states is given in Figs. 5a and $5 \mathrm{~b}$, which illustrate the changes in the energy spectrum versus the vector $k_{\|\|}$variation along the path shown in Fig. 3 for the structures SC $\{111\}$ and FCC $\{111\}$, respectively. For $k_{\|}$from the centre of the zone ( $\Gamma$-point) there are no localized states in the system (all are within the band). With $k_{\|}$approaching the point $K$ at first one state emerges and is then followed by another one. At the point $K$ they reach the farthest distance from the border of the band (in the energy scale) and thus become the most strongly localized (measure by the value of $t$, in this case, equal to $\infty$ ). Convergence of the band to a point is a consequence of the $\left|\Gamma_{1}^{k} \|\right|$ reaching zero. This fact also entails other consequences, which are, however, of no importance to us. Returning to the $\Gamma$-point (the path on the zone becomes closed) the states are subsequently made to enter the band and finally the system is in the same conditions as at the beginning.

a)

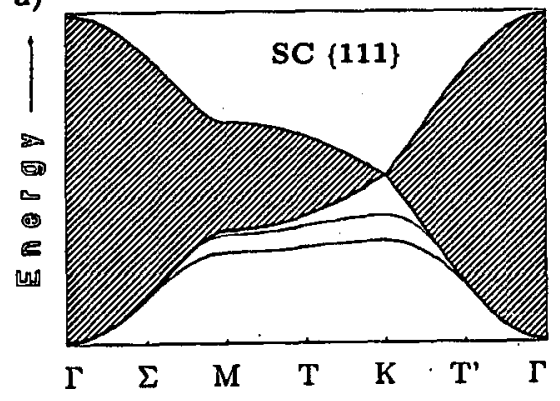

b)

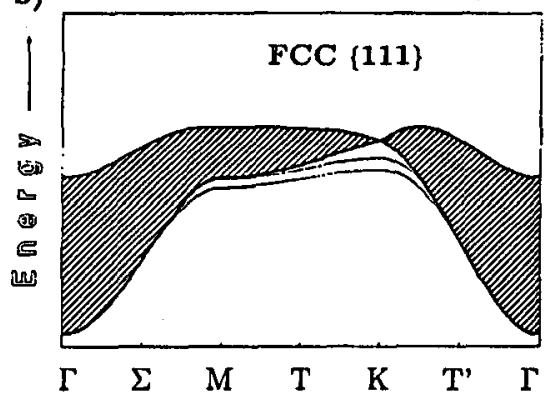

Fig. 5. Changes in the energy of surface states for SC $\{111\}$ structure versus the in-plane vector $k_{\|}$(varying along the path of Fig. 3) (a). The same as (a) but for FCC \{111\} structure (b). The difference between (a) and (b) is only apparently great; this is only due to the presence of the $\Gamma_{0}^{k} \|$ term in the dispersion relation, which equals 0 in the first case and differs from zero in the latter thus resulting in a kind of modulation of the energy diagram. However, considering the values of $k_{\|}$for which surface states appear and disappear we find that both situations are alike. Values assumed are: $L=21$, $A_{1}=0.8, A_{2}=0.5$.

The difference between Fig. $5 \mathrm{a}$ and $5 \mathrm{~b}$ is only apparently great; this is simply due to the presence in the dispersion relation (37) of the $\Gamma_{0}^{k_{\|} \|}$term, which equals 0 in the former case and differs from zero in the latter case, thus resulting in a kind of modulation of the energy diagram. However, considering the values of $\boldsymbol{k}_{\|}$for which surface states appear and disappear we find that both situations are alike. This confirms our observation that the structural function really important for our model is only $\left|\Gamma_{1}^{k_{\|}}\right|$and the knowledge of its explicit form is of much help in the investigation of particular structures. Probably the form of this function depends 
on the type of the two-dimensional Bravais lattice obtained as the result of the cut-out. This problem requires further investigation. As there are only five lattices of this kind, it may be expected that the number of $\left|\Gamma_{1}^{k_{\|}}\right|$functions (function types) will be slightly more than five. The table included in the previous section of this paper seems to support this view.

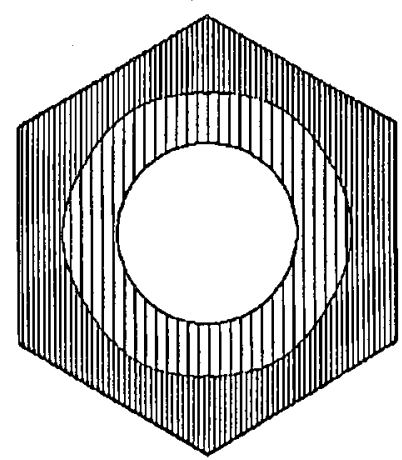

Fig. 6. Two-dimensional Brillouin zone with regions of existence of surface states indicated. Shading used here is taken from Fig. 1. Note that the greatest number of surface states occurs in the vicinity of the zeros of $\left|\Gamma_{1}^{k_{\|}}\right|$and that the boundaries of the respective regions of existence of localized states result from the shape of the surface in Fig. 2. The parameter values used in plotting Fig. 6 are those of Fig. 5.

The advantage of knowing the $\left|\Gamma_{1}^{k} \|\right|$ function is illustrated in Fig. 6 where the areas of surface states occurrence on the Brillouin zone are shown for the same parameters as those assumed for the case shown in Fig. 5. While constructing these areas we have made use of the fact that the borderlines between them are the cross-section lines of the relevant structural function; the determination of the $\left|\Gamma_{1}^{k} \|\right|$ values for which a localized state appears for fixed parameters $A_{1}, A_{2}, L$ is not difficult provided that we use the pinning diagram and the relations (40) and (42).

Were we to pose the problem in a more general form, i.e., for arbitrary $A_{1}$, $A_{2}$ and $L$, we might expect to get interesting patterns as the shape of the $\left|\Gamma_{1}^{k_{\|}}\right|$ surface for the structure considered is rather complicated.

Reference to the pinning diagram considerably facilitates the classification of the patterns obtained because a pair $A_{1}$ and $A_{2}$ determines uniquely the point $(R, P)$ on the diagram by means of the relations

$$
R=\frac{A_{1} A_{2}-1}{A_{1} A_{2}+1}, \quad P=\frac{A_{1}+A_{2}}{A_{1} A_{2}+1} .
$$

The diagram may be divided into fragments which correspond to topologically undistinguishable patterns on the Brillouin zone, i.e., the areas may differ in size but not in shape for all pairs $\left(A_{1}, A_{2}\right)$ for which the $(R, P)$ points (they determine via Eq. (54)) lie within a given fragment. This fact is illustrated in Fig. 7 showing the part of the diagram corresponding to the acoustic states within the marked 


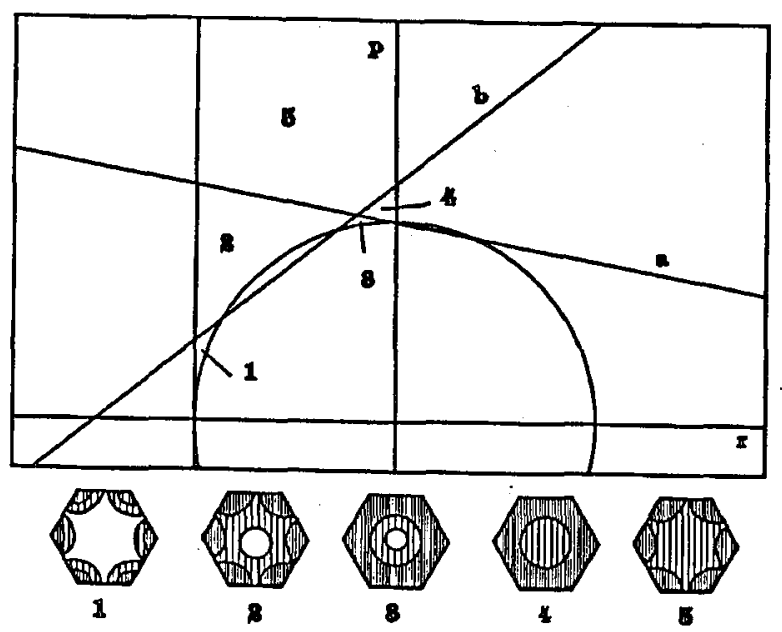

Fig. 7. Connection between the pinning diagram and the patterns on the 1st Brillouin zone. Taking initial parameters $r$ and $p$ from the regions labelled 1-5, we get corresponding (or topologically undistinguishable) patterns on the zone, as indicated below in the diagram. We have chosen only a small part of the $r-p$ plane because the whole number of possible patterns is about 50. The straight lines marked on the figure fulfill the following equations:

line a: $p=(-1 / L) r+1$; line b: $p=[-(4 L-5) / 3 L] r+(5 L-4) / 3 L$.

shapes of the areas of surface state occurrence on the zones. Thus within a given fragment of the diagram the areas may change in size but not in shape. The way in which variations of the parameters affect the size of the area on the zone is an interesting problem but we shall not consider it in detail as it would require a thorough discussion of the pinning diagram as carried out in Ref. [2].

\section{Conclusions}

In this work we have considered a thin magnetic film described by a Heisenberg Hamiltonian within the model of surface inhomogeneity. Of particular interest to us was the appearance of surface spin-wave states induced by changes of a dynamic quantity, and we have found that the wave vector in the plane of the film $\left(k_{\| 1}\right)$ may actually be responsible for the appearance of surface states in our model. Moreover, for chosen crystal orientations the areas of surface state occurrence in two-dimensional Brillouin zones have been determined.

Irrespective of the physical interpretation of the above considerations it is worth noting that the mathematics developed in this work can be applied to other problems concerning, for example, phonons or electrons. 


\section{Acknowledgment}

The authors are indebted to the Committee for Scientific Research for their support under the grant No. 223229102.

\section{References}

[1] H. Puszkarski, Acla Phys. Pol. A 38, 217 (1970).

[2] H. Puszkarski, Surf. Sci. 34, 125 (1973).

[3] S.V. Tiablikov, Metody kvanlovoy teorii magnetizma, Nauka, Moskva 1975 (in Russian).

[4] R.J. Jelitto, Z. Nat.forsch. A 19, 1567 (1964).

[5] R.J. Jelitto, Z. Nat.forsch. A 19, 1580 (1964). 